\title{
Dedicated service improves the accuracy of Barrett's oesophagus surveillance: a prospective comparative cohort study
}

\section{James Britton, ${ }^{1,2}$ Kelly Chatten, ${ }^{3}$ Tom Riley, ${ }^{4}$ Richard R Keld, ${ }^{1}$ Shaheen Hamdy, ${ }^{2,4}$ John McLaughlin, ${ }^{2,4}$ Yeng Ang ${ }^{2,4}$}

\begin{abstract}
- Additional material is published online only. To view, please visit the journal online (http://dx.doi.org/10.1136/ bmjres-2018-101019).

\section{${ }^{1}$ Department of} Gastroenterology, Wrightington, Wigan and Leigh NHS Foundation Trust, Wigan, UK

${ }^{2}$ Division of Diabetes, Endocrinology and Gastroenterology, School of Medical Sciences, Faculty of Biology, Medicine and Health, The University of Manchester, Manchester, UK

${ }^{3}$ Department of Gastroenterology, Stockport NHS Foundation Trust, Stockport, Stockport, UK

${ }^{4}$ Department of

Gastroenterology, Salford Royal NHS Foundation Trust, Salford, UK
\end{abstract}

\section{Correspondence to}

Dr Yeng Ang, Department of Gastroenterology, Salford Royal NHS Foundation Trust, Salford M6 8HD, UK; Yeng.Ang@srft. nhs.uk

Received 17 May 2018 Revised 16 July 2018 Accepted 19 August 2018 Published Online First 5 September 2018

\section{Check for updates}

(C) Author(s) (or their employer(s)) 2019. No commercial re-use. See rights and permissions. Published by BMJ.

To cite: Britton J,

Chatten K, Riley T, et al.

Frontline Gastroenterology

2019;10:128-134.

\section{ABSTRACT}

Objectives Standards for Barrett's oesophagus (BO) surveillance in the UK are outlined in the British Society of Gastroenterology (BSG) guidelines. This study aimed to assess the quality of current surveillance delivery compared with a dedicated service.

Design All patients undergoing $\mathrm{BO}$ surveillance between January 2016 and July 2017 at a single National Health Service district general hospital were included. Patients had their endoscopy routed to a dedicated $\mathrm{BO}$ endoscopy list or a generic service list. Prospective data were analysed against the BSG guidelines and also compared with each patient's prior surveillance endoscopy.

Results 361 patients were scheduled for surveillance of which 217 attended the dedicated list, 78 attended the non-dedicated list and 66 did not have their endoscopy. The dedicated list adhered more closely to the BSG guidelines when compared with the non-dedicated and prior endoscopy, respectively; Prague classification ( $100 \%$ vs $87.3 \%$ vs $82.5 \%, p<0.0001)$, hiatus hernia delineation ( $100 \%$ vs $64.8 \%$ vs $63.3 \%$, $\mathrm{p}<0.0001)$, location and number of biopsies recorded $(99.5 \%$ vs $5.6 \%$ vs $6.9 \%, p<0.0001)$, Seattle protocol adherence ( $72 \%$ vs $42 \%$ vs $50 \%, \mathrm{p}<0.0001)$ and surveillance interval adherence (dedicated 100\% vs prior endoscopy $75 \%, \mathrm{p}<0.0001)$. Histology results from the dedicated and non-dedicated list cohorts revealed similar rates of intestinal metaplasia $(79.8 \%$ vs $73.1 \%, p=0.12$ ) and dysplasia/oesophageal adenocarcinoma ( $4.3 \%$ vs $2.6 \%, p=0.41$ ).

Conclusions The post-BSG guideline era of BO surveillance remains suboptimal in this UK hospital setting. A dedicated service appears to improve the accuracy and consistency of surveillance care, although the clinical significance of this remains to be determined.

\section{INTRODUCTION}

The incidence of oesophageal adenocarcinoma (OAC) in the Western world is increasing. ${ }^{2}$ Once invasive, this cancer harbours a poor prognosis ${ }^{3} 4$ and limited treatment options. Barrett's oesophagus (BO) has been firmly recognised as a precursor to OAC with a European general population prevalence reported at $1.3 \%-1.6 \% 0^{5} 6$ and an annual cancer conversion rate of $0.33 \%$ in a recent meta-analysis. ${ }^{7}$ Over the last 30 years, there have been significant research gains in the attempt to diminish the progression of $\mathrm{BO}$ to OAC. In particular, retrospective cohort and comparative studies suggest that endoscopic surveillance correlates with earlier staging and improved cancer survival. ${ }^{8-14}$ This has culminated into the development of national surveillance guidelines and dysplasia treatment pathways in Europe and the USA. ${ }^{15-18}$

Although endoscopic surveillance is widely practised, it remains a controversial topic with no published randomised controlled trials supporting its efficacy, ${ }^{19}$ and therefore uncertainty remains about best practice. Indeed, this area was ranked number 4 in the top 10 research priorities for future BO and gastro-oesophageal reflux disease research in a recent UK-wide exercise which engaged both patients and healthcare providers. ${ }^{20}$ The latest British Society of Gastroenterology (BSG) guidelines provided clearer diagnostic criteria, defined surveillance intervals and a minimum endoscopic dataset for reporting. ${ }^{15}$ In the absence 
of conclusive evidence for the use of advanced imaging modalities, they also advocate high-definition white light endoscopy with the Seattle biopsy protocol. ${ }^{21}$ Preguideline UK data suggest that patients with $\mathrm{BO}$ have received inconsistent care from perhaps less well-informed or disengaged physicians. ${ }^{22}$ The aim of this study was to define current care in the postguideline era and to judge whether a dedicated $\mathrm{BO}$ list performs better in terms of BSG guidance metrics and compliance than a non-dedicated list in a typical National Health Service (NHS) hospital setting.

\section{Ethical considerations}

This piece of work incorporated elements of both service evaluation and clinical audit defined by the NHS Health Research Authority ${ }^{23}$; therefore, formal ethical approval was not required.

\section{DESIGN AND SETTING}

All patients with BO surveillance between January 2016 and July 2017 at a single NHS district general hospital in the UK were included in the cohort for analysis. The majority of patients enrolled in surveillance were identified prospectively via the endoscopy booking department or at endoscopy. Patients underwent their surveillance endoscopy on a dedicated BO list or a non-dedicated endoscopy list. This routing process was not randomised or influenced by the study team and occurred purely due to endoscopy capacity and patient availability on dates they were offered their test. We prospectively collected data against the BSG dataset for endoscopy reporting (see online supplementary material) while also recording the number of biopsies taken, histology results and appropriateness of surveillance intervals. Prospective surveillance

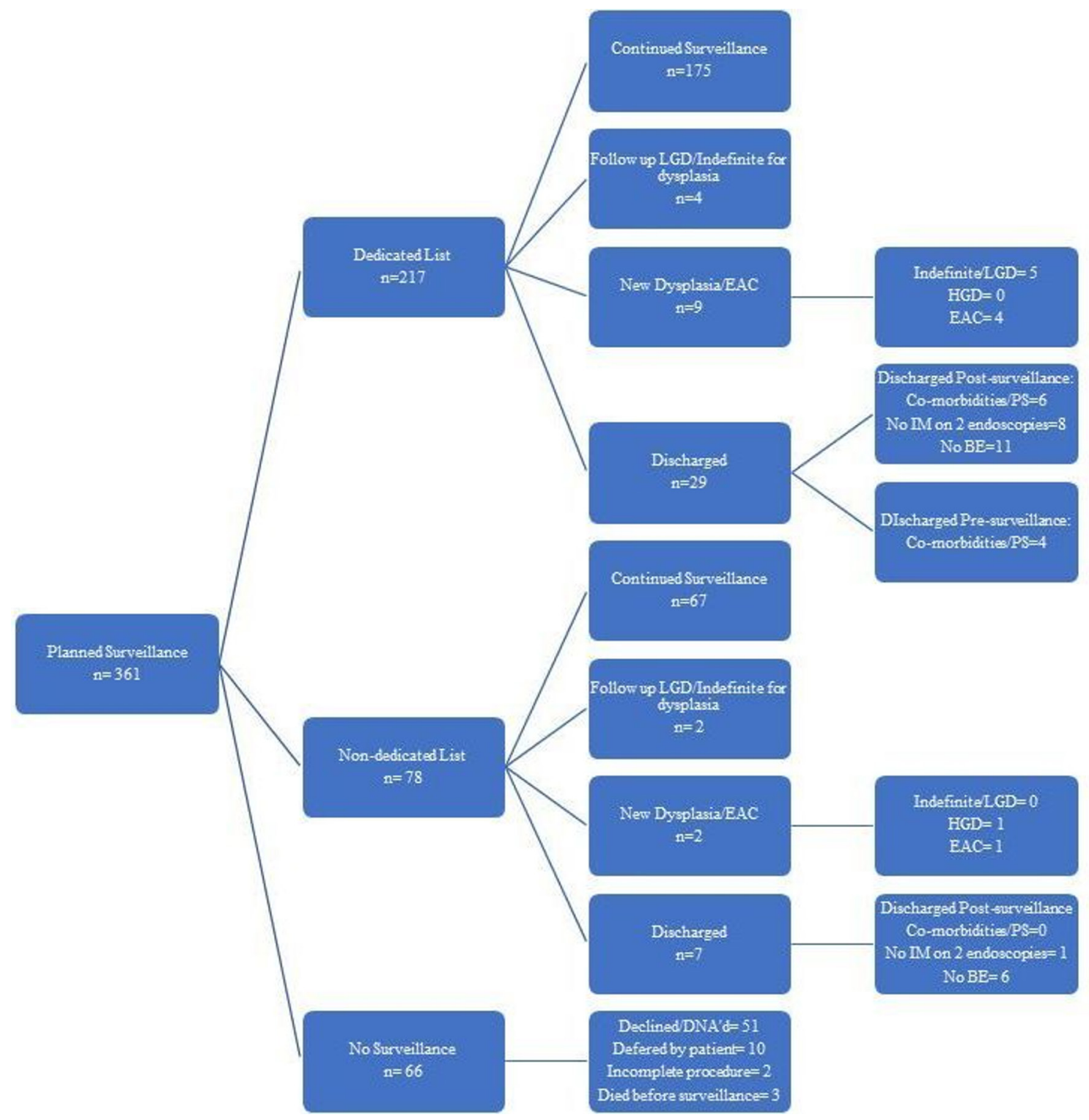

Figure 1 BO surveillance January 2016 to July 2017. BO, Barrett's oesophagus; HGD, high-grade dysplasia; IM, intestinal metaplasia; LGD, lowgrade dysplasia; OAC, oesophageal adenocarcinoma; PS, performance status. 
data were then compared with each patient's previous surveillance endoscopy. Data are expressed as mean \pm SD and percentiles unless otherwise stated. Fisher's exact test was used for comparison of means. A p value of $<0.05$ was taken to show statistical significance.

\section{RESULTS}

\section{Patient groups and demographics}

Three hundred sixty-one patients were scheduled for BO surveillance between January 2016 and July 2017. Of these, 217 attended a dedicated BO list, 78 had a generic service list and 66 did not have their endoscopy completed (figure 1). Both surveillance groups had comparable demographics in terms of age, sex, Prague classification and comorbidity prevalence (see online supplementary material).

\section{Endoscopists}

The dedicated list was conducted by a single endoscopist with a specialist interest in BO. By comparison, patients attending the non-dedicated lists $(n=78)$ and retrospective lists $(n=229)$ had their endoscopy undertaken by a range of general endoscopists. Nurse endoscopists (39\%) and consultant gastroenterologists $(37 \%)$ conducted the most procedures, followed by specialty trainees (13\%) and consultant surgeons (11\%). In total, there were 20 (prospective) and 35 (retrospective) individual healthcare professionals providing surveillance for these non-dedicated cohorts, respectively, with an average of 2.3 and 4.1 surveillance procedures per endoscopist per year.

\section{Endoscopy reporting}

Patients who were discharged from surveillance were excluded from the prospective cohorts prior to analysis of endoscopy reporting (dedicated list $n=188$, non-dedicated list $n=71$, total $n=259$ ). In addition, the previous endoscopy reports of all 259 patients were retrospectively reviewed to provide a historic comparison of service provision. Of the 259 retrospective reports, 14 were excluded as their prior endoscopy predated the BSG guideline publication, and a further 16 were excluded as their reports were unavailable. The dedicated BO endoscopy list achieved significantly greater adherence to the BSG guideline for endoscopy reporting when compared with both the non-dedicated and retrospective cohorts. The prevalence of visible lesions documented was comparable between the cohorts; however, there were significantly more Barrett's islands reported on the dedicated list. This likely reflects the enhanced reporting seen in the dedicated cohort than an actual difference in cohort prevalence (table 1).

\section{Other quality indicators}

Adherence to the Seattle protocol was significantly greater in the dedicated cohort when calculated on a case-by-case basis (table 2). However, this did not translate into significantly higher rates of intestinal metaplasia (table 2) or dysplasia (table 3). Overall discharge rates were also unaffected by the type of list employed (table 3). Interestingly, it appears that more patients were discharged from the dedicated list due to comorbidities or performance status $(n=10,34.5 \%$ of discharges) compared with none in the non-dedicated list. Patients in the non-dedicated cohort $(\mathrm{n}=78)$ were more likely to be discharged $(n=7)$ from a consultant-led list $(n=6 / 7)$ than a nurse-led list $(n=0 / 7)$ despite conducting a comparable number of procedures (30 consultant led and 31 nurse led).

Within the same time frame, 197 new BO diagnoses were referred to the service for consideration of surveillance. After further assessment, a large proportion of

\begin{tabular}{|c|c|c|c|c|c|}
\hline & $\begin{array}{l}\text { Dedicated BO } \\
\text { surveillance } \\
\text { endoscopy, } \mathrm{n}=188\end{array}$ & $\begin{array}{l}\text { Non-dedicated } \\
\text { surveillance } \\
\text { endoscopy, n=71 }\end{array}$ & $P$ values & $\begin{array}{l}\text { Retrospective } \\
\text { previous BO } \\
\text { surveillance, } n=229\end{array}$ & $\begin{array}{l}\text { P values } \\
\text { (dedicated vs } \\
\text { retrospective) } \\
\end{array}$ \\
\hline Standards & $\begin{array}{l}\text { January } \\
2016 \text { to July } 2017\end{array}$ & $\begin{array}{l}\text { January } \\
2016 \text { to July } 2017\end{array}$ & $\begin{array}{l}\text { (dedicated vs } \\
\text { non-dedicated) }\end{array}$ & $\begin{array}{l}\text { November } \\
2013 \text { to June } 2016\end{array}$ & \\
\hline BO length (Prague classification) & $100 \%(n=188)$ & $87.3 \%(n=62)$ & $P<0.0001$ & $82.5 \%(n=189)$ & $P<0.0001$ \\
\hline $\begin{array}{l}\text { BO island description (distance from incisors } \\
\text { and length) }\end{array}$ & $96.6 \%(n=28)$ & $0 \%(n=0)$ & $P<0.0001$ & $17.6 \%(n=3)$ & $P<0.0001$ \\
\hline $\mathrm{BO}$ island prevalence & $15.4 \%(n=29)$ & $5.6 \%(n=4)$ & $P=0.02$ & $7.4 \%(n=17)$ & $P=0.0074$ \\
\hline Hiatus hernia documentation & $100 \%(n=188)$ & $64.8 \%(n=46)$ & $P<0.0001$ & $63.3 \%(n=145)$ & $P<0.0001$ \\
\hline $\begin{array}{l}\text { Visible lesion documentation } \\
\text { (yes or no) }\end{array}$ & $100 \%(n=188)$ & $94.4 \%(n=67)$ & $P=0.005$ & $89.9 \%(n=206)$ & $P<0.0001$ \\
\hline Visible lesion prevalence & $9.6 \%(n=18)$ & $8.5 \%(n=6)$ & $P=0.50$ & $4.8 \%(n=11)$ & $P=0.0436$ \\
\hline $\begin{array}{l}\text { Visible lesion description } \\
\text { (distance from incisors+Paris classification) }\end{array}$ & $94.4 \%(n=17)$ & $0 \%(n=0)$ & $P<0.0001$ & $0 \%(n=0)$ & $P<0.0001$ \\
\hline $\begin{array}{l}\text { Biopsies } \\
\text { (location and number taken) }\end{array}$ & $99.5 \%(n=187)$ & $5.6 \%(n=4)$ & $P<0.0001$ & $6.9 \%(n=16)$ & $P<0.0001$ \\
\hline
\end{tabular}

BO, Barrett's oesophagus. 
Table 2 Other endoscopy quality indicators

\begin{tabular}{|c|c|c|c|c|c|}
\hline & $\begin{array}{l}\text { Dedicated BO } \\
\text { surveillance } \\
\text { endoscopy, } n=188\end{array}$ & $\begin{array}{l}\text { Non-dedicated } \\
\text { surveillance } \\
\text { endoscopy, n=71 }\end{array}$ & P values & $\begin{array}{l}\text { Retrospective } \\
\text { previous BO } \\
\text { surveillance, } n=229\end{array}$ & P values \\
\hline Standards & $\begin{array}{l}\text { January } \\
2016 \text { to July } 2017\end{array}$ & $\begin{array}{l}\text { January } \\
2016 \text { to July } 2017 \\
\end{array}$ & $\begin{array}{l}\text { (dedicated vs } \\
\text { non-dedicated) }\end{array}$ & $\begin{array}{l}\text { November } \\
2013 \text { to June } 2016\end{array}$ & $\begin{array}{l}\text { (dedicated vs } \\
\text { retrospective) }\end{array}$ \\
\hline Average no of biopsies (histology reported) & 7.5 & 6 & & 6.3 & \\
\hline Average Prague (M) & 3.6 & 4.1 & & 4.1 & \\
\hline $\begin{array}{l}\text { Seattle protocol adherence \% } \\
\text { (case-by-case }{ }^{*} \text { ) }\end{array}$ & $\begin{array}{l}72 \% \\
(n=135 / 188)\end{array}$ & $\begin{array}{l}42 \% \\
(n=26 / 62)\end{array}$ & $P<0.0001$ & $\begin{array}{l}50 \% \\
(n=94 / 189)\end{array}$ & $P<0.0001$ \\
\hline Intestinal metaplasia & $\begin{array}{l}79.80 \% \\
(n=150)\end{array}$ & $\begin{array}{l}73.10 \% \\
(n=51)\end{array}$ & $P=0.12$ & $\begin{array}{l}79.90 \% \\
(n=183)\end{array}$ & $P=0.532$ \\
\hline $\begin{array}{l}\text { Surveillance interval } \\
\text { appropriate }\end{array}$ & $\begin{array}{l}100 \% \\
(n=188)\end{array}$ & Nat & & $\begin{array}{l}75 \% \\
(n=147)\end{array}$ & $P<0.0001$ \\
\hline
\end{tabular}

*Expected Seattle biopsy number (Prague $M / 2 \times 4$ ) versus number of biopsies taken.

tDuring this time frame, endoscopists referred to the dedicated BO service to arrange.

BO, Barrett's oesophagus.

these $(n=60,30.5 \%)$ were not enrolled into surveillance (the reasons are documented in table 3 ).

\section{DISCUSSION}

The major finding from this study was the enhanced adherence to current standards of care achieved by the implementation of a dedicated BO service list, rather than unmanaged allocation of patients with BO to any clinician undertaking endoscopy in mixed, unselected lists. In particular, improved endoscopic reporting in terms of $\mathrm{BO}$ delineation: Prague classification, hiatus hernia and island descriptions. Adherence to surveillance intervals was also improved when compared with the retrospective data collected. This would potentially prevent over surveillance in many cases as the majority of patients with inappropriate surveillance intervals had short segment disease with a 2 -year interval $(88 \%, n=42 / 48)$. The dedicated service

\begin{tabular}{|c|c|c|c|}
\hline & $\begin{array}{l}\text { Dedicated BO surveillance endoscopy } \\
(\mathrm{n}=217)^{*}\end{array}$ & $\begin{array}{l}\text { Non-dedicated surveillance endoscopy } \\
(\mathrm{n}=78)^{*}\end{array}$ & $P$ values \\
\hline \multicolumn{4}{|l|}{ New dysplasia diagnoses } \\
\hline Indefinite/LGD & 5 & 0 & \\
\hline$H G D$ & 0 & 1 & \\
\hline OAC & 4 & 1 & \\
\hline Total & 9 & 2 & \\
\hline Diagnosis rate $\dagger$ & $4.3 \%$ & $2.6 \%$ & $P=0.41$ \\
\hline \multicolumn{4}{|l|}{ Discharges from surveillance } \\
\hline Discharged pre endoscopy & 4 (comorbidities/PS) & 0 & \\
\hline Discharged post endoscopy & $\begin{array}{l}\text { Comorbidities } / P S=6 \\
\text { No } I M \text { on two endoscopies }=8 \\
\text { No } B O=11\end{array}$ & $\begin{array}{l}\text { Comorbidities } / \mathrm{PS}=0 \\
\text { No IM on two endoscopies=1 } \\
\text { No } \mathrm{BO}=6\end{array}$ & \\
\hline Total discharged & 29 & 7 & \\
\hline Total discharge rate $\%$ & $13.4 \%$ & $9.0 \%$ & $P=0.21$ \\
\hline \multicolumn{4}{|l|}{ Discharges of new referrals } \\
\hline New diagnoses referred & 197 & & \\
\hline Enrolled in surveillance & 137 & & \\
\hline Discharged for no surveillance & $\begin{array}{l}60 \\
\text { (comorbidities/PS=23) } \\
\text { No IM on two endoscopies=28 } \\
\text { No BO on reassessment=9 }\end{array}$ & & \\
\hline New diagnosis discharge rate & $30.5 \%$ & & \\
\hline \multicolumn{4}{|c|}{$\begin{array}{l}\text { *These numbers include those who were discharged after their endoscopy. } \\
\text { †This calculation excludes LGD and indefinite for dysplasia follow-up patients ( } n=4 \text { for dedicated list, } n=2 \text { for non-dedicated list) and patients dischar } \\
\text { pre-endoscopy ( } n=4 \text { for dedicated list). } \\
\text { BO, Barrett's oesophagus; HGD, high-grade dysplasia; IM , intestinal metaplasia; LGD, low-grade dysplasia; OAC, oesophageal adenocarcinoma; PS, } \\
\text { performance status. }\end{array}$} \\
\hline
\end{tabular}


also discharged $13.4 \%$ of patients from surveillance, in particular patients with significant comorbidities or poor performance status. These discharge rates are comparable to historic UK data from a specialist centre $(11 \%) .^{24}$ These findings probably reflect a vested interest of the BO service provider when compared with a general endoscopist who is often just the 'technician' of surveillance, probably unaware of the detailed guidance. This is echoed by the lack of discharges, seen in this study, by nurse endoscopists, which is an important consideration when planning future surveillance care pathways that may be nurse led. The dedicated service also used a $\mathrm{BO}$ clinic as a platform to have more informed discussions about surveillance appropriateness. This is useful when one considers the number of patients enrolled at a time when diagnostic criteria were less clear. ${ }^{25}$ The main use of the BO clinic, however, was for consultation of newly diagnosed patients. This clinic provided an opportunity to ratify the diagnosis and assess the appropriateness of surveillance in terms of patient fitness and willingness. This service appears most valuable as a high proportion $(n=60,30.5 \%)$ of patients may have been inappropriately or automatically enrolled in longer-term surveillance without review. This could cause undue patient burden and impact on endoscopy provisions. It is unclear from this study what proportion of this assessment and decision making would have happened without the service in place.

Although not found to be statistically significant, this study suggests that a dedicated BO surveillance list may diagnose dysplasia more readily than those attending other 'ad hoc' lists during the same time frame $(4.3 \%$ vs $2.6 \%)$. There are a number of potential reasons for this. First, limiting the number of endoscopists conducting surveillance may allow them to become more experienced and proficient in identifying and sampling abnormal areas. Second, the dedicated BO service was also limited to seven gastroscopies per session. Current recommendations from the joint advisory group on gastrointestinal endoscopy are a maximum of $210 \mathrm{~min}$ per endoscopy list which crudely equates to 10 gastroscopies or 5 colonoscopies per list. ${ }^{26}$ This adjusted allotted time for surveillance procedures ensures adequate time for mucosal inspection and Seattle biopsies, particularly in long-segment disease. Although oesophageal withdrawal time was not documented, previous research suggests a Barrett's inspection time of greater than $1 \mathrm{~min}$ per centimetre detects more endoscopically suspicious lesions $(54.2 \%$ vs $13.3 \%, \mathrm{p}=0.04)$ and higher rates of highgrade dysplasia and OAC $(40.2 \%$ vs $6.7 \%, \mathrm{p}=0.06) .{ }^{27}$ This is now reflected in the most recent publication of Quality Standards in upper gastrointestinal endoscopy. ${ }^{28}$ However, further clarification is needed as this recommendation is based on a post hoc analysis of a single clinical trial. Finally, the dedicated list achieved significantly greater compliance to the Seattle protocol.
Logically, one would expect low adherence to biopsy protocols to be associated with lower dysplasia detection rates. ${ }^{29}$ One would also expect a greater number of biopsies to yield significantly higher rates of intestinal metaplasia (IM), ${ }^{30}$ which was not demonstrated in this study. A larger sample size would be needed to confidently delineate whether there is a significant difference in IM and dysplasia rates between these groups.

\section{Limitations}

Limitations of this study include: retrospective in design which can be subject to bias; relatively small numbers, from a single centre and over a short time frame, choosing only a single guideline to measure quality standards and the possible effects of patient factors in this non-randomised study. However, despite these limitations, the findings appear both consistent

\section{Significant of this study}

What is already known on this topic

- Barrett's oesophagus (BO) surveillance, outlined in the British Society of Gastroenterology (BSG) guidelines, is widely practised in the UK.

- Surveillance remains a controversial topic among endoscopists with no randomised controlled trial demonstrating its efficacy.

- Historic, preguideline, data suggest that BO surveillance has been inconsistent.

- The potential role of a dedicated BO surveillance service has been identified as a top 10 research priority in a recent exercise which engaged both patients and healthcare providers.

\section{What this study adds}

- BO surveillance remains suboptimal in the post-BSG guideline era. In particular, endoscopic reporting, adherence to the Seattle protocol biopsies and appropriateness of surveillance intervals.

- A dedicated BO service improves the accuracy and consistency of surveillance care in line with current best practice.

\section{How might it impact on clinical practice in the} foreseeable future

- This study demonstrates that standards can be met if a service manages its patients proactively. This may be reproduced in other National Health Service hospitals.

- A higher powered, multicentre study is required to determine if these gains translate into better clinical and patient-centred outcomes, such as dysplasia diagnosis rates and health-related quality of life.

- In time, a dedicated service may provide a more stable transition to future care pathways. For example, BO individual risk stratification models or enhanced endoscopic surveillance techniques. 
with the literature and show that standards can be met if the clinical service manages its patients proactively.

\section{CONCLUSIONS}

The right patient undergoing the right test at the right time is a mantra which applies to all surveillance strategies. This study demonstrates that a dedicated service can ratify the cohort of surveyed patients (right patient), conduct a more consistent test in line with current best practice (right test) and ensure appropriate surveillance intervals (right time). Without addressing current inadequacies, our ability to move forward and provide future care pathways will be hampered. In time, a dedicated service may provide a more stable transition to future guidelines, for example, easier adaptation to individual risk stratification models and advanced endoscopic techniques. From this study, it remains unclear whether such a service can consistently improve clinical outcomes, such as dysplasia diagnosis rates. Further prospective, higher powered, multicentre studies are needed to evaluate these potential clinical gains alongside patient-centred outcomes, such as health-related quality of life, disease-specific knowledge and overall satisfaction with services. ${ }^{31}$

Contributors All authors significantly contributed to this work. The concept and design of this study was instigated by YA, RRK, JB, JM and SH. Data were collected by TR and JB. KC led the data analysis. JB and $\mathrm{KC}$ drafted the initial manuscript. All authors had a role in the writing and revision of the manuscript prior to submission.

Funding The authors have not declared a specific grant for this research from any funding agency in the public, commercial or not-for-profit sectors.

Competing interests None declared.

Patient consent Not required.

Provenance and peer review Not commissioned; externally peer reviewed.

Author note The abstract has been accepted for a poster presentation at the BSG conference in June 2018.

\section{REFERENCES}

1 Lepage $\mathrm{C}$, Rachet B, Jooste V, et al. Continuing rapid increase in esophageal adenocarcinoma in England and Wales. Am J Gastroenterol 2008;103:2694-9.

2 Thrift AP, Whiteman DC. The incidence of esophageal adenocarcinoma continues to rise: analysis of period and birth cohort effects on recent trends. Ann Oncol 2012;23:3155-62.

3 Cancer survival in england adults diagnosed. $2013 \mathrm{https}: / /$ www.ons.gov.uk/peoplepopulationandcommunity/ healthandsocialcare/conditionsanddiseases/bulletins/cancersu rvivalinenglandadultsdiagnosed/2013-10-29.

4 Eloubeidi MA, Mason AC, Desmond RA, et al. Temporal trends (1973-1997) in survival of patients with esophageal adenocarcinoma in the United States: a glimmer of hope? Am J Gastroenterol 2003;98:1627-33.

5 Zagari RM, Fuccio L, Wallander MA, et al. Gastro-oesophageal reflux symptoms, oesophagitis and Barrett's oesophagus in the general population: the Loiano-Monghidoro study. Gut 2008;57:1354-9.

6 Ronkainen J, Aro P, Storskrubb T, et al. Prevalence of Barrett's esophagus in the general population: an endoscopic study. Gastroenterology 2005;129:1825-31.
7 Desai TK, Krishnan K, Samala N, et al. The incidence of oesophageal adenocarcinoma in non-dysplastic Barrett's oesophagus: a meta-analysis. Gut 2012;61:970-6.

8 Corley DA, Levin TR, Habel LA, et al. Surveillance and survival in Barrett's adenocarcinomas: a population-based study. Gastroenterology 2002;122:633-40.

9 Fountoulakis A, Zafirellis KD, Dolan K, et al. Effect of surveillance of Barrett's oesophagus on the clinical outcome of oesophageal cancer. Br J Surg 2004;91:997-1003.

10 Rubenstein JH, Sonnenberg A, Davis J, et al. Effect of a prior endoscopy on outcomes of esophageal adenocarcinoma among United States veterans. Gastrointest Endosc 2008;68:849-55.

11 Cooper GS, Yuan Z, Chak A, et al. Association of prediagnosis endoscopy with stage and survival in adenocarcinoma of the esophagus and gastric cardia. Cancer 2002;95:32-8.

12 Streitz JM, Andrews CW, Ellis FH. Endoscopic surveillance of Barrett's esophagus. Does it help? J Thorac Cardiovasc Surg 1993;105:383-7; discussion 387-8.

13 van Sandick JW, van Lanschot JJB, Kuiken BW, et al. Impact of endoscopic biopsy surveillance of Barrett's oesophagus on pathological stage and clinical outcome of Barrett's carcinoma. Gut 1998;43:216-22.

14 Peters JH, Clark GW, Ireland AP, et al. Outcome of adenocarcinoma arising in Barrett's esophagus in endoscopically surveyed and nonsurveyed patients. $J$ Thorac Cardiovasc Surg 1994;108:813-21; discussion 821-2.

15 Fitzgerald RC, di Pietro M, Ragunath K, et al. British Society of Gastroenterology guidelines on the diagnosis and management of Barrett's oesophagus. Gut 2014;63:7-42.

16 Weusten B, Bisschops R, Coron E, et al. Endoscopic management of Barrett's esophagus: European Society of Gastrointestinal Endoscopy (ESGE) Position Statement. Endoscopy 2017;49:191-8.

17 Bennett C, Moayyedi P, Corley DA, et al. BOB CAT: A Large-Scale Review and Delphi Consensus for Management of Barrett's Esophagus With No Dysplasia, Indefinite for, or Low-Grade Dysplasia. Am J Gastroenterol 2015;110:662-82.

18 Shaheen NJ, Falk GW, Iyer PG, et al. ACG Clinical Guideline: Diagnosis and Management of Barrett's Esophagus. Am J Gastroenterol 2016;111:30-50.

19 Old O, Moayyedi P, Love S, Team TBT, et al. Barrett's Oesophagus Surveillance versus endoscopy at need Study (BOSS): protocol and analysis plan for a multicentre randomized controlled trial. J Med Screen 2015;22:158.

20 Britton J, Gadeke L, Lovat L, et al. Research priority setting in Barrett's oesophagus and gastro-oesophageal reflux disease. Lancet Gastroenterol Hepatol 2017;2:824-31.

21 Levine DS, Blount PL, Rudolph RE, et al. Safety of a systematic endoscopic biopsy protocol in patients with Barrett's esophagus. Am J Gastroenterol 2000;95:1152-7.

22 Mandal A, Playford RJ, Wicks AC. Current practice in surveillance strategy for patients with Barrett's oesophagus in the UK. Aliment Pharmacol Ther 2003;17:1319-24.

23 Health Research Authority. http://www.hra-decisiontools.org. uk/research/docs/DefiningResearchTable_Oct2017-1.pdf. 2017.

24 Anagnostopoulos GK, Pick B, Cunliffe R, et al. Barrett's esophagus specialist clinic: what difference can it make? Diseases of the Esophagus 2006;19:84-7.

25 Sharma P, Morales TG, Sampliner RE. Short segment Barrett's esophagus--the need for standardization of the definition and of endoscopic criteria. Am J Gastroenterol 1998;93:1033-6.

26 Valori R, 2012 How many 'points' should there be on an endoscopy list? www.thejag.org.uk.

27 Gupta N, Gaddam S, Wani SB, et al. Longer inspection time is associated with increased detection of high-grade dysplasia and esophageal adenocarcinoma in Barrett's esophagus. Gastrointest Endosc 2012;76:531-8.

28 Beg S, Ragunath K, Wyman A, et al. Quality standards in upper gastrointestinal endoscopy: a position statement of the 
British Society of Gastroenterology (BSG) and Association of Upper Gastrointestinal Surgeons of Great Britain and Ireland (AUGIS). Gut 2017;66:1886-99.

29 Abrams JA, Kapel RC, Lindberg GM, et al. Adherence to biopsy guidelines for Barrett's esophagus surveillance in the community setting in the United States. Clin Gastroenterol Hepatol 2009;7:736-42.
30 Harrison R, Perry I, Haddadin W, et al. Detection of intestinal metaplasia in Barrett's esophagus: an observational comparator study suggests the need for a minimum of eight biopsies. Am J Gastroenterol 2007;102:1154-61.

31 Britton J, Keld R, Prasad N, et al. Effect of diagnosis, surveillance, and treatment of Barrett's oesophagus on healthrelated quality of life. Lancet Gastroenterol Hepatol 2018;3. 